\title{
Electron energy loss in carbon nanostructures
}

\author{
A. Rivacoba ${ }^{1,2}$ and F. J. García de Abajo $^{2, *}$ \\ ${ }^{1}$ Materialen Fisika Saila, Kimika Fakultatea, UPV/EHU, 1072 Posta Kutxa, 20080 Donostia, Spain \\ ${ }^{2}$ Donostia International Physics Center (DIPC) and Centro Mixto CSIC-UPV/EHU, Apartado 1072, 20080 San Sebastián, Spain
}

(Received 10 October 2002; published 21 February 2003)

\begin{abstract}
The response of fullerenes and carbon nanotubes is investigated by representing each carbon atom by its atomic polarizability. The polarization of each carbon atom produces an induced dipole that is the result of the interaction with a given external field plus the mutual interaction among carbon atoms. The polarizability is obtained from the dielectric function of graphite after invoking the Clausius-Mossotti relation. This formalism is applied to the simulation of electron-energy-loss spectra both in fullerenes and in carbon nanotubes. The case of broad electron beams is considered and the loss probability is analyzed in detail as a function of the electron deflection angle within a fully quantum-mechanical description of the electrons. A general good agreement with available experiments is obtained in a wide range of probe energies between $1 \mathrm{keV}$ and $60 \mathrm{keV}$.
\end{abstract}

DOI: 10.1103/PhysRevB.67.085414

PACS number(s): 79.20.Uv, 73.22.Lp, 73.61.Wp

\section{INTRODUCTION}

Since the discovery of fullerenes, ${ }^{1}$ the collective excitations of these molecules have received considerable attention. The first electron-energy-loss spectra (EELS) of solid $\mathrm{C}_{60}$ were reported by Saito and co-workers. ${ }^{2}$ Later, EELS have been employed to study fullerenes supported on surfaces, ${ }^{3,4}$ single-walled fullerenes in the gas phase, ${ }^{5,6}$ multishell fullerenes, ${ }^{7-10}$ and other carbon structures of more exotic shapes. ${ }^{11-16}$ Along with this experimental effort, theoretical studies of the electronic structure and the collective excitations of these molecules have also been carried out. ${ }^{17-20}$ In particular, Saito and co-workers ${ }^{2}$ noted the strong similarity between loss spectra of fullerenes and graphite, and pointed out that this was due to the fact that the electronic structure of both of these materials consisted of $\sigma$ orbitals along the carbon bonds and $\pi$ orbitals in the normal direction. This idea has been exploited by Lucas et al. ${ }^{7}$ to describe the response of fullerenes in connection with EELS. They used a continuum model consisting of a classical dielectric sphere that was made of a nonisotropic dielectric function equal to that of graphite. Henrard and Lambin ${ }^{21}$ extended that work by using the discrete-dipole approximation $^{22,23}$ (DDA), where the fullerenes were considered as a system of coupled point dipoles, and their atomic polarizability tensor was obtained from the dielectric function of graphite via the Clausius-Mossotti relation.

The continuum model $^{7}$ works well for multishell fullerenes, where the thickness of the target can be properly assigned, but this is not the case with single-layer clusters, where the thickness parameter becomes critical, while it is not well defined. However, the DDA provides a reasonable description in the latter case.

So far, EELS electrons have been described as classical charged particles, ${ }^{21}$ although such an approach presents some disadvantages, connected to the fact that the energy-loss spectra depend strongly on the beam position relative to the specific atomic positions of the carbon cluster, and, in particular, the loss probability diverges when the beam intersects any of the atomic positions, a fact that derives from the unphysical singularity at the origin of the dipole potential $\left(\sim 1 / r^{2}\right)$ used in this approach. This pathological behavior at small distances is connected to unphysically large values of the momentum transfer, which are included implicitly in the classical energy-loss theory. ${ }^{24,25}$ The classical theory is then only justified for relatively distant collisions, where the contribution of large momentum transfer is negligible. Another drawback of a classical description of the probe derives from its inability to account for the momentum-transfer dependence of the loss probability, a magnitude that is accessible to the EELS theory ${ }^{26}$ and experiments ${ }^{8,10,16}$ via the selection of electron-scattering angles: momentum-resolved EELS have actually proved to be a useful tool for obtaining optical and electronic properties in many cases. ${ }^{8,10,16}$ One more issue related to momentum transfer (in this case with the component parallel to the electron velocity) is the so-called recoil of the electron, which can play a noticeable role at low electron energies such as those employed in some experiments with fullerenes in order to avoid damage of the specimen. ${ }^{24}$

In this work, we make use of the self-energy formalism to calculate the energy-loss probability within a fully quantummechanical description of the electron, while the response of the carbon cluster is expressed in terms of the screened interaction as calculated in the DDA via the atomic polarizability of the carbon atoms. The energy-loss probability is obtained as a function of both the lost energy and the electron deflection angle (i.e., the momentum-transfer component normal to the electron beam). This solves the problems that come about in a classical description of the electron, as discussed above: divergences in close encounters are avoided in a natural way and the contribution of electron recoil is fully accounted for. For the electron energies under consideration, the initial and final electron states can be described by plane waves. This is a very realistic approach to EELS experiments when a broad beam is employed. Furthermore, when narrow beams of $\sim 0.5 \mathrm{~nm}$ are used, one can interpret our extendedbeam results as spectra averaged over the impact parameter ${ }^{24}$ which raises the question of the suitability of the dipole potential $\sim 1 / r^{2}$ near the atoms; nevertheless closeencounter processes correspond to large momentum transfers, and, therefore, they contribute little when calculating the spectra of electrons collected with a small scattering 
angle, as is usually done in the experiments. Notice that for classical electrons, inelastic scattering occurs up to an impact parameter $b \leqslant v / \omega$, where $v$ is the electron velocity and $\omega$ is the energy-loss, so that the broad beam approach is likely to overestimate the weight of low-energy excitations for target sizes comparable to the lateral coherence length of the electron beam.

The screened interaction calculated in Sec. II is actually the Green function of Poisson's equation in the presence of the sample, and thus, it provides the response of the fullerene to any arbitrary external field. When we apply this theory to simulate EELS in single-layered carbon structures (Sec. V), very good agreement with experiments is obtained, indicating that the screened interaction could equally be applied to other problems involving the response of fullerenes with the inclusion of atomic details (e.g., in scanning tunnel microscopy, light absorption, ${ }^{27}$ van der Waals forces, ${ }^{28}$ and image states $^{29}$ ). A similar approach could be also applied to other materials such as boron nitride structures.

Atomic units (a.u., i.e., $\hbar=m=e=1$ ) will be used throughout this work, unless otherwise specified.

\section{THE SCREENED INTERACTION OF A FINITE ATOMIC CLUSTER}

We shall consider a system composed by $N$ atoms located at the positions $\mathbf{r}^{a}$, with $a=1, \ldots, N$. Each atom can be polarized so that it behaves like an induced dipole. The polarizability tensor of atom $a$ will be denoted by $\boldsymbol{\alpha}^{a}(\omega)$ in frequency space $\omega$. When an external electric field $\mathbf{E}(\omega)$ is applied to the system, the induced dipole moment $\mathbf{p}^{a}(\omega)$ of atom $a$ is the response to the total field, that is, the external field plus the field induced by the rest of the dipoles. One finds

$$
p_{i}^{a}=\sum_{j k} \alpha_{i j}^{a}\left[E_{j}\left(\mathbf{r}^{a}, \omega\right)+\sum F_{j k}^{a b} p_{k}^{b}\right],
$$

where the indices $i, j$, and $k$ refer to Cartesian coordinates. The second term on the right hand side of Eq. (1) involves the dipole-dipole interaction, which can be written in terms of the relative atomic positions $\mathbf{r}^{a b}=\mathbf{r}^{a}-\mathbf{r}^{b}$ as

$$
F_{j k}^{a b}=\frac{1}{\left(r^{a b}\right)^{3}}\left[3 \frac{r_{j}^{a b} r_{k}^{a b}}{\left(r^{a b}\right)^{2}}-\delta_{j k}\right], \quad a \neq b=0, \quad a=b .
$$

Notice that matrix $F$ is fully symmetric both in coordinate indices $i j$ and in atomic ones $a b$. From Eq. (1), the dipolar moments of the system are found to be

$$
p_{i}^{a}(\omega)=\sum_{b, j k}\left[M^{-1}\right]_{i j}^{a b} \alpha_{j k}^{b} E_{k}\left(\mathbf{r}^{b}, \omega\right),
$$

where $M^{-1}$ is the inverse of the $\omega$-dependent matrix

$$
M_{i j}^{a b}=\delta^{a b} \delta_{i j}-\alpha_{i k}^{a} F_{k j}^{a b} .
$$

Equation (3) permits calculating the induced-dipole moments for an arbitrary external field, from which one can in turn obtain the induced field. In particular, this permits to derive the so-called screened interaction of the system $W\left(\mathbf{r}, \mathbf{r}^{\prime}, \omega\right)$, which is the solution of the Poisson equation for a point charge at $\mathbf{r}^{\prime}$, that is,

$$
\nabla^{2} W\left(\mathbf{r}, \mathbf{r}^{\prime}, \omega\right)=-4 \pi \delta\left(\mathbf{r}-\mathbf{r}^{\prime}\right) .
$$

In this case, the field $\mathbf{E}(\mathbf{r})$ created by the external probe is just the Coulomb field $1 /\left|\mathbf{r}-\mathbf{r}^{\prime}\right|$, and, therefore, the induced part of the screened interaction can be straightforwardly written as

$$
W^{\text {ind }}\left(\mathbf{r}, \mathbf{r}^{\prime}, \omega\right)=-\sum_{a b, i k} C_{i k}^{a b}(\omega) \frac{\left(r_{i}-r_{i}^{a}\right)}{\left|\mathbf{r}-\mathbf{r}^{a}\right|^{3}} \frac{\left(r_{k}^{\prime}-r_{k}^{b}\right)}{\left|\mathbf{r}^{\prime}-\mathbf{r}^{b}\right|^{3}},
$$

where

$$
C_{i k}^{a b}(\omega)=\sum_{j}\left[M^{-1}\right]_{i j}^{a b} \alpha_{j k}^{b} .
$$

Like $M$ above, this matrix is symmetric in both sets of indices, so that $W^{\text {ind }}\left(\mathbf{r}, \mathbf{r}^{\prime}, \omega\right)$ is also symmetric with respect to the spatial variables $\mathbf{r}$ and $\mathbf{r}^{\prime}$.

From a practical point of view, it is important to note that the dependence on frequency and spatial variables is separated in this formalism: the dependence on $\omega$ is fully contained in matrix $C$, whereas the dependence on both $\mathbf{r}$ and $\mathbf{r}^{\prime}$ comes from the last two factors in Eq. (6). Therefore, although the actual dipoles have to be calculated numerically to obtain matrix $C$, the spatial-dependent part can be dealt with analytically in many cases. For instance, it will be useful to rewrite Eq. (6) in momentum space as

$$
\begin{aligned}
W^{\text {ind }}\left(\mathbf{r}, \mathbf{r}^{\prime}, \omega\right)= & \frac{4}{(2 \pi)^{4}} \sum_{a b, i k} C_{i k}^{a b}(\omega) \\
& \times \iint d \mathbf{p} d \mathbf{q} \frac{q_{i} p_{k}}{\mathbf{p}^{2} \mathbf{q}^{2}} e^{\mathrm{i} \mathbf{q} \cdot\left(\mathbf{r}-\mathbf{r}^{a}\right)} e^{i \mathbf{p} \cdot\left(\mathbf{r}^{\prime}-\mathbf{r}^{b}\right)} .
\end{aligned}
$$

The response of the system to any external probe can be now expressed in terms of Eq. (6). As an example, the energy-loss experienced by a fast classical electron is obtained as a double integral of the screened interaction, ${ }^{25}$ which can be solved analytically ${ }^{30}$ to recover expressions (18)-(20) of Ref. 21.

\section{ENERGY LOSS OF FAST ELECTRONS IN A SYSTEM OF COUPLED DIPOLES}

As an application of the formalism presented above, we now proceed to compute the energy-loss experienced by a broad beam of fast electrons incoming along the $z$ axis into an atomic cluster, described as a set of coupled induced dipoles. We study the transition between initial and final states of the electrons, described by plane waves $\Psi_{0}(\mathbf{r})$ $=V^{-1 / 2} e^{i \mathbf{k}_{0} \cdot \mathbf{r}}$ and $\Psi_{f}(\mathbf{r})=V^{-1 / 2} e^{i \mathbf{k}_{f} \cdot \mathbf{r}}$, respectively, where $\mathbf{k}_{0}$ and $\mathbf{k}_{f}$ are the initial and final momenta, and $V$ is the normalization volume. The transition probability can be written $^{33,34}$ as 


$$
\begin{aligned}
P(\omega)= & \frac{2}{v} \sum_{f} \int d \mathbf{r} d \mathbf{r}^{\prime} \Psi_{f}^{*}\left(\mathbf{r}^{\prime}\right) \Psi_{f}(\mathbf{r}) \operatorname{Im}\left\{-W_{\text {ind }}\left(\mathbf{r}, \mathbf{r}^{\prime}, \omega\right)\right\} \\
& \times \Psi_{0}^{\star}(\mathbf{r}) \Psi_{0}\left(\mathbf{r}^{\prime}\right) \delta\left(\omega-\omega_{0 f}\right),
\end{aligned}
$$

where $\mathbf{v}=\mathbf{k}_{0}$ is the velocity of the incoming probe, taken to be along the positive $z$ axis, $\omega_{0 f}=\frac{1}{2}\left(\mathbf{k}_{0}^{2}-\mathbf{k}_{f}^{2}\right)$ is the transition energy, and $\operatorname{Im}\left\{W^{\text {ind }}\left(\mathbf{r}, \mathbf{r}^{\prime}, \omega\right)\right\}$ is the imaginary part of the screened interaction. The sum over the final states $f$ can be transformed into an integral over the momentum transfer $\mathbf{Q}$ $=\mathbf{k}_{0}-\mathbf{k}_{f}$. One finds

$$
\begin{aligned}
P(\omega)= & -\frac{2}{v} \frac{1}{(2 \pi)^{3}} \frac{1}{V} \int d \mathbf{Q} \int d \mathbf{r} d \mathbf{r}^{\prime} e^{-i \mathbf{Q} \cdot\left(\mathbf{r}-\mathbf{r}^{\prime}\right)} \\
& \times \operatorname{Im}\left\{W^{\text {ind }}\left(\mathbf{r}, \mathbf{r}^{\prime}, \omega\right)\right\} \delta\left(\omega-\mathbf{Q v}+\frac{1}{2} Q^{2}\right) .
\end{aligned}
$$

Now, inserting Eq. (8) into this expression, one can write

$$
\begin{aligned}
P(\omega)= & \frac{4}{\pi v} \sum_{a b, k j} \operatorname{Im}\left\{C_{k j}^{a b}(\omega)\right\} \int d \mathbf{Q} \frac{Q_{j} Q_{k}}{Q^{4}} e^{-i \mathbf{Q} \cdot\left(\mathbf{r}^{b}-\mathbf{r}^{a}\right)} \\
& \times \delta\left(\omega-\mathbf{Q} \cdot \mathbf{v}+\frac{1}{2} Q^{2}\right)
\end{aligned}
$$

The probability of collecting an electron after suffering an energy-loss $\omega$ and being scattered inside a circular aperture of half-angle $\theta_{m}$ can be expressed as the sum of the contributions of excitations characterized by $\omega$ and $\mathbf{q}$ [the energy and the component of the momentum transfer normal to the beam direction, respectively, that is, $\left.\mathbf{Q}=\left(\mathbf{q}, q_{z}\right)\right]$ as follows:

$$
P(\omega)=-\frac{8}{v^{2}} \sum_{a b, k j} \operatorname{Im}\left\{C_{k j}^{a b}(\omega)\right\} \Gamma_{j k}^{a b},
$$

where $\Gamma_{j k}^{a b}$ is a matrix (symmetric in both $a b$ and $i j$ ) that reduces to

$$
\begin{gathered}
\Gamma_{11}^{a a}=\Gamma_{22}^{a a}=\frac{1}{2} \int_{0}^{q_{m}} d q \frac{q^{3}}{\Delta}, \\
\Gamma_{33}^{a a}=\int_{0}^{q_{m}} d q \frac{q q_{z}^{2}}{\Delta}, \\
\Gamma_{i j}^{a a}=0 i \neq j,
\end{gathered}
$$

when $a=b$. Here, the $z$ component of the momentum transfer, $q_{z}$, and $\Delta$ are both functions of $\omega$ and $q$, that is,

$$
\begin{gathered}
q_{z}=v-\sqrt{v^{2}-2 \omega-q^{2}}, \\
\Delta=\left(q^{2}+q_{z}^{2}\right)^{2} \sqrt{1-\frac{2 \omega+q^{2}}{v^{2}}},
\end{gathered}
$$

and $q_{m} \sim v \theta_{m}$ is the largest possible value of $q$. The matrix elements coupling different atoms can be written as

$$
\begin{aligned}
\Gamma_{11}^{a b}= & \int_{0}^{q_{m} d q} \frac{d}{\Delta} \cos \left[q_{z}\left(z^{b}-z^{a}\right)\right] q^{2}\left[q \cos ^{2} \theta_{a b} J_{0}\left(q \rho_{a b}\right)\right. \\
& \left.-\rho_{a b}^{-1} \cos \left(2 \theta_{a b}\right) J_{1}\left(q \rho_{a b}\right)\right] \\
\Gamma_{22}^{a b}= & \int_{0}^{q_{m}} \frac{d q}{\Delta} \cos \left[q_{z}\left(z^{b}-z^{a}\right)\right] q^{2}\left[q \sin ^{2} \theta_{a b} J_{0}\left(q \rho_{a b}\right)\right. \\
& \left.+\rho_{a b}^{-1} \cos \left(2 \theta_{a b}\right) J_{1}\left(q \rho_{a b}\right)\right] \\
\Gamma_{33}^{a b}= & \int_{0}^{q_{m}} \frac{d q}{\Delta} \cos \left[q_{z}\left(z^{b}-z^{a}\right)\right] q_{z}^{2} q J_{0}\left(q \rho_{a b}\right), \\
\Gamma_{12}^{a b}=- & \frac{1}{2} \int_{0}^{q_{m}} \frac{d q}{\Delta} \cos \left[q_{z}\left(z^{b}-z^{a}\right)\right] \sin \left(2 \theta_{a b}\right) q^{3} J_{2}\left(q \rho_{a b}\right), \\
\Gamma_{13}^{a b}= & -\int_{0}^{q_{m}} \frac{d q}{\Delta} \sin \left[q_{z}\left(z^{b}-z^{a}\right)\right] \cos \theta_{a b} q_{z} q^{2} J_{1}\left(q \rho_{a b}\right), \\
\Gamma_{23}^{a b}= & -\int_{0}^{q_{m} \frac{d q}{\Delta}} \sin \left[q_{z}\left(z^{b}-z^{a}\right)\right] \sin \theta_{a b} q_{z} q^{2} J_{1}\left(q \rho_{a b}\right),
\end{aligned}
$$

where $J_{n}$ is the Bessel functions of first kind and order $n$, $\rho_{a b}=\sqrt{\left(x^{a}-x^{b}\right)^{2}+\left(y^{a}-y^{b}\right)^{2}}$, and $\theta_{a b}=\cos ^{-1}\left[\left(x^{b}-x^{a}\right) / \rho_{a b}\right]$.

The expression (14) for $q_{z}$ derives from the $\delta$ function ensuring energy conservation in Eq. (11), where the term $\frac{1}{2} Q^{2}$ represents the recoil of the electron. For large electron velocities, this term is negligible and $q_{z}$ can be approximated by the classical value $q_{z}=\omega / v$. Actually, Ritchie ${ }^{24}$ proved that even when all the scattered electrons were collected, the effect of this term is small for fast $(100 \mathrm{keV})$ electrons.

The coupling between different atoms in Eq. (12) is introduced via the screened interaction, where the polarization of neighboring atoms is strongly correlated. This leads to the existence of polarization waves that play the same role as plasmons in extended media. The spatial extension of these waves in the direction normal to the beam can be analyzed by studying the dependence of the $\Gamma_{i j}^{a b}$ integrals (see above) on the separation between dipoles in the direction normal to the electron beam, $\rho_{a b}$, and for the sake of obtaining an estimate one can use the classical-approach approximations consisting in neglecting the recoil (i.e., $q_{z}=\omega / v$ ) and extending the integral in $q$ from zero to infinity for $a \neq b$; one finds

$$
\begin{aligned}
\Gamma_{33}^{a b} & \rightarrow\left\{\frac{\omega}{v}\right\}^{2} \cos \left[\frac{\omega}{v}\left(z^{b}-z^{a}\right)\right] \int_{0}^{\infty} d q \frac{q J_{0}\left(q \rho_{a b}\right)}{\left(q^{2}+\frac{\omega_{2}}{v}\right)^{2}} \\
& =\frac{\omega \rho_{a b}}{2 v} \cos \left[\frac{\omega}{v}\left(z^{b}-z^{a}\right)\right] K_{0}\left(\frac{\omega \rho_{a b}}{v}\right)
\end{aligned}
$$

where $K_{0}$ is the modified Bessel function, and similar expressions involving $K_{m}$ are found for the rest of the $\Gamma_{i j}^{a b}$ integrals. Taking into account the asymptotic behavior of $K_{m}$, Eq. (16) permits us to say that the dipoles are effectively coupled up to a distance of the order of the adiabatic 
length $v / \omega$, similar to the lateral extension of the charge density associated with surface plasmons in extended media. $^{31}$

Equation (12) can be applied to any atomic cluster described by the atomic polarizabilities. In the case of a system of noninteracting dipoles [i.e, when $F_{j k}^{a b}$ is set to 0 in Eq. (4)], assuming an isotropic polarizability, $\alpha_{j k}^{a}=\alpha \delta_{j k}$, and neglecting the recoil, the energy-loss per atom probability reduces to

$$
P(\omega)=\frac{4}{v^{2}} \sum_{a} \operatorname{Im}\{-\alpha(\omega)\} \ln \left[1+\left(\frac{q_{m} v}{\omega}\right)^{2}\right]
$$

This expression reproduces the energy-loss probability of a system of uncoupled dielectric spheres of radius $R$, where the polarizability is given by $\alpha=(\epsilon-1) /(\epsilon+2) R^{3}$, in the limit of small momentum transfer, $q R \ll 1 .^{32,33,35}$ This means that the suitability of this approach is restricted to values $q_{m}$ $\leqslant 1 / R$, where $R$ has to be understood as the radius of a region containing the valence electrons. For $\sigma$ electrons one can estimate $R$ as half the distance between $\mathrm{C}$ atoms in graphite (i.e., $1.42 \AA$ ), which limits the validity of the present theory to $q_{m} \leqslant 1$ a.u. Notice that this limit is related only to the suitability of the dipolar potential near the origin: as one reaches the limit $q R \sim 1$, higher multipolar excitations become more relevant than the dipolar one, and one should rely on a multipole approach. ${ }^{36}$ This fact, pointed out by Keller and Coplan, ${ }^{5}$ possesses another limit to the applicability of the DDA theory.

Although it has been proved ${ }^{25}$ that classical energy-loss expressions can be recovered from quantum-mechanical ones by neglecting recoil and taking the $q_{m} \rightarrow \infty$ limit, such an expression is not defined in the present context, since diagonal terms of $\Gamma$ diverge logarithmically at large values of $q$, which is another manifestation of the unphysical behavior of the dipolar potential near the target-atom nuclei.

\section{THE SCREENED INTERACTION OF INFINITE SYSTEMS: CARBON NANOTUBES}

The theory presented in the preceding sections can be straightforwardly applied to finite atomic clusters, such as fullerenes, but is not suitable to study infinite systems. In what follows, we will recast this theory to describe a carbon nanotube consisting of the infinite repetition of a unit cell of length $d$ along the direction of the $x$ axis. In order to take advantage of the translational invariance of this system, we write the coupling integrals $F_{i j}^{a b}$ as

$$
F_{i j}^{a b}=\int_{-\infty}^{\infty} d q e^{i q\left(x^{a}-x^{b}\right)} \phi_{i j}^{a b}(q),
$$

where $\phi_{i j}^{a b}(q)$ is the Fourier transform of $F_{i j}^{a b}$, which reduces to

$$
\phi_{11}^{a b}(q)=-\frac{1}{\pi} q^{2} K_{0}\left(|q| \rho_{a b}\right)
$$

$$
\begin{gathered}
\phi_{1 i}^{a b}(q)=-\frac{i}{\pi} q|q| K_{1}\left(|q| \rho_{a b}\right) \frac{\left(r_{i}^{a}-r_{i}^{b}\right)}{\rho_{a b}}, \\
\phi_{i j}^{a b}(q)=\frac{1}{\pi}\left\{q^{2} K_{0}\left(|q| \rho_{a b}\right) \frac{\left(r_{i}^{a}-r_{i}^{b}\right)\left(r_{j}^{a}-r_{j}^{b}\right)}{\rho_{a b}^{2}}\right. \\
\left.-|q| K_{1}\left(|q| \rho_{a b}\right)\left[\frac{\delta_{i j}}{\rho_{a b}}-2 \frac{\left(r_{i}^{a}-r_{i}^{b}\right)\left(r_{j}^{a}-r_{j}^{b}\right)}{\rho_{a b}^{3}}\right]\right\},
\end{gathered}
$$

with $i, j=2,3 \quad(j=1$ refers to the $x$ direction $)$ and $\rho_{a b}$ $=\sqrt{\left(y^{a}-y^{b}\right)^{2}+\left(z^{a}-z^{b}\right)^{2}}$. Notice that $\phi_{i j}^{a b}(q)$ depends only on the components of the relative position vector that are normal to the translational axis of symmetry, and, therefore, it is enough to compute it for the atoms of a given unit cell of the tube.

For atoms located in equivalent positions of the tube (i.e., $\rho_{a b}=0$ ), only the diagonal elements are involved, but obviously, the above expressions diverge as

$$
\phi_{11}^{a b}=-2 \phi_{22}^{a b}=-2 \phi_{33}^{a b}=\frac{1}{\pi} \int_{-\infty}^{\infty} d x \frac{e^{-i q x}}{|x|^{3}} .
$$

However, this divergence can be avoided on physical grounds by excluding the self-interaction (i.e., an atoms interaction with itself) from the summation over target atoms [see Eq. (2)]. In a similar way, one can write

$$
\left[M^{-1}\right]_{i j}^{a b}=\int_{0}^{g} d q e^{i q\left(x^{a}-x^{b}\right)} \nu_{i j}^{a b}(q),
$$

where $g=2 \pi / d$ is the reciprocal lattice constant and $\nu_{i j}^{a b}(q)$ satisfies the secular equation

$$
\frac{1}{g} \delta_{i l} \delta^{a c}=\sum_{b, k j}\left\{\delta_{i j} \delta^{a b}-\alpha_{i k}^{a} R_{k j}^{a b}(q)\right\} \nu_{j l}^{b c}(q) .
$$

Here, the indices $a, b$, and $c$ run only over the first unit cell and $R_{k j}^{a b}(q)$ is defined as

$$
R_{k j}^{a b}(q)=g \sum_{n}\left[e^{i n g\left(x^{a}-x^{b}\right)} \phi_{k j}^{a b}(q+n g)\right]
$$

For $a=b$ (equivalent atoms), the $n=0$ term must be excluded from Eq. (23) in order to avoid the self-interaction.
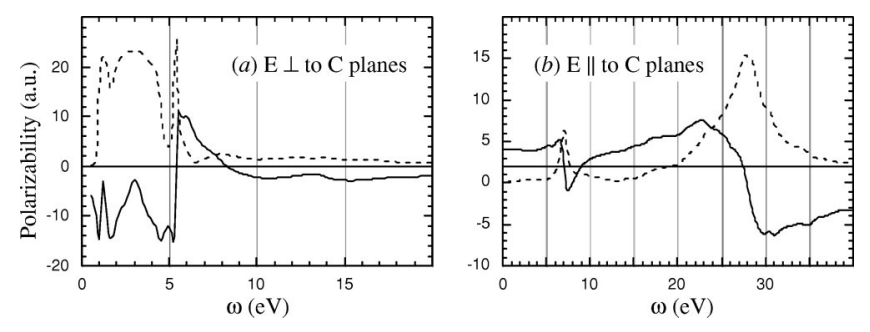

FIG. 1. Polarizability of a carbon atom in graphite for the electric field perpendicular (a) and parallel (b) to the atomic planes. The solid (dashed) curves stand for the real (imaginary) part of the polarizability. 

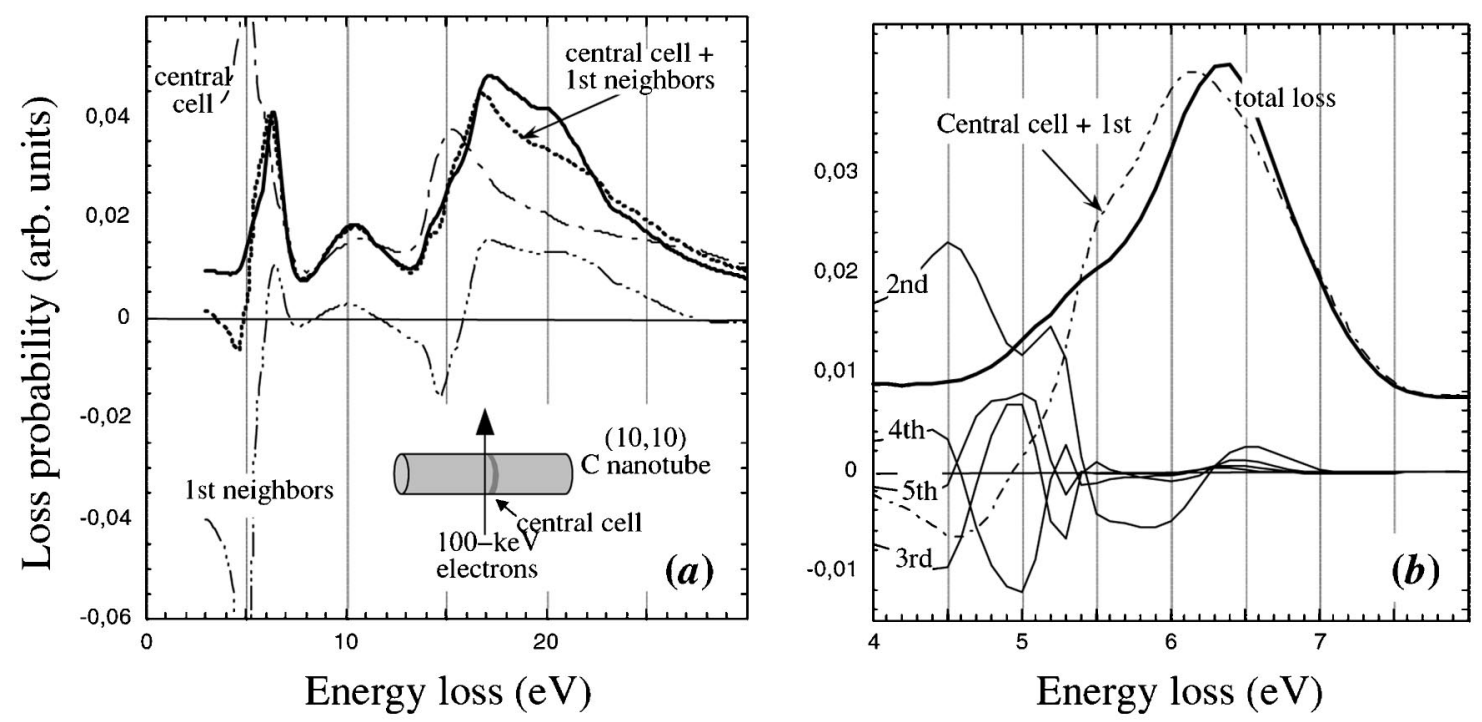

FIG. 2. (a) Energy-loss probability per carbon atom in a $(10,10)$ carbon nanotube. The primary electron energy is $100 \mathrm{keV}$ and the collector aperture half-angle is $\theta_{c}=1 \mathrm{mrad}$. The solid curve represents the total energy-loss probability, while the rest of the curves stand for the partial contribution of the central cell, the first neighboring cells, and the central cell plus the first neighboring cells (see labels and discussion in the text). (b) Detail of the low-energy region of the spectrum in (a), showing separate contributions of up to the fifth neighboring cells.

This removes the noted divergence from Eq. (20) and allows using finite numbers in the secular equation (22). Finally, one finds

$$
R_{11}^{a a}(q)=-2 R_{22}^{a a}(q)=-2 R_{33}^{a a}(q)=\frac{4}{d^{3}} \sum_{n>0} \frac{\cos (n q d)}{n^{3}}
$$

which is a well-defined sum. The fact that $R_{k j}^{a b}(q)$ is a periodic function of period $g$ has been exploited to reduce the integration interval in Eq. (18) to the first Brillouin zone in Eq. (21). Once the matrix $\nu(q)$ has been obtained, $M^{-1}$ can be computed via Eq. (21) and the problem is solved.

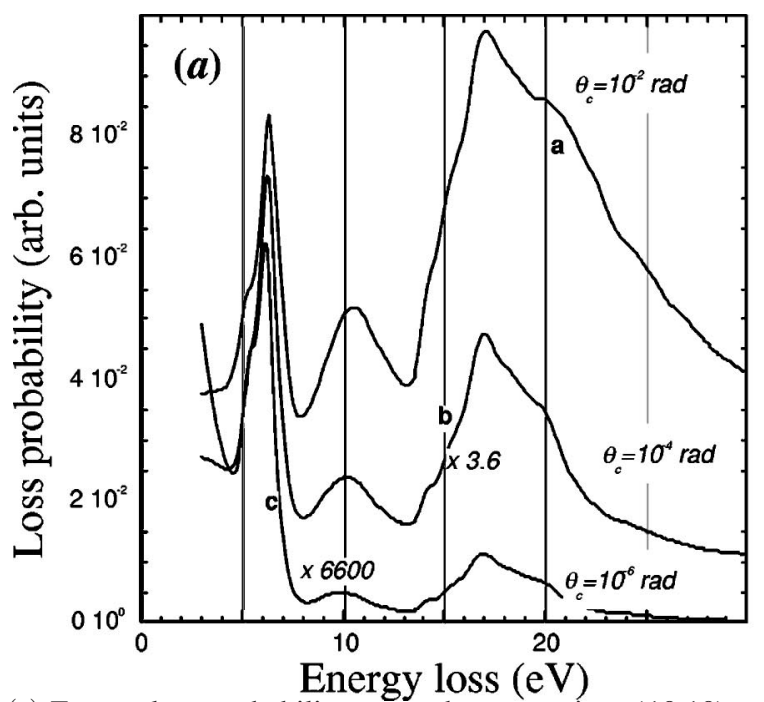

\section{RESULTS AND DISCUSSION}

In order to apply this theory to fullerenes, one must obtain first the polarizability of the $\mathrm{C}$ atoms in the molecule. This is done via the Clausius-Mosotti relation by using the bulk dielectric function of graphite, ${ }^{37}$ assuming that the polarizability of $\mathrm{C}$ atoms is essentially the same in graphite and in fullerenes owing to their similar local atomic environment. In Fig. 1, we plot both the real and the imaginary parts of the two independent components of the polarizability tensor (i.e., normal and parallel to the graphite planes). These plots correspond to $\mathrm{C}$ atoms in a graphitelike structure and have no physical meaning unless the coupling with neighboring atoms is considered. It is interesting to note that both com-

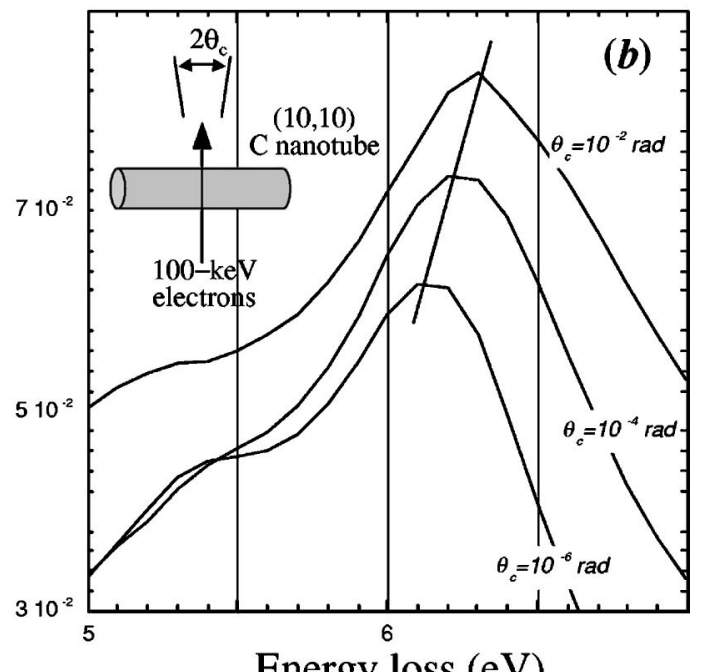

Energy loss (eV)

FIG. 3. (a) Energy-loss probability per carbon atom in a $(10,10)$ carbon nanotube for three different collector aperture half-angles (see labels). The primary electron energy is $100 \mathrm{keV}$. (b) Detail of the $\pi$-plasmon peak in (a) showing a positive dispersion with increasing aperture size. 


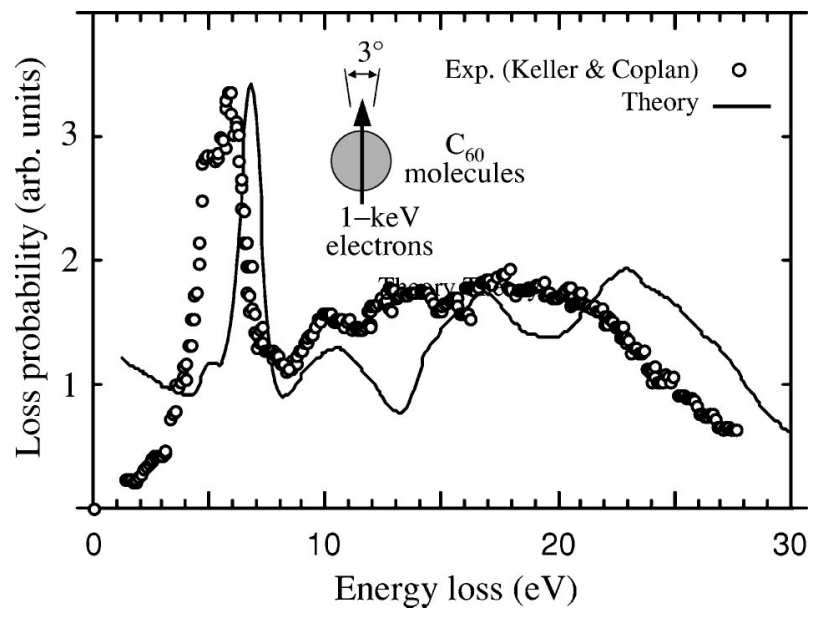

FIG. 4. Comparison between theory (solid curve) and experiment (circles, taken from Ref. 5) for the energy-loss probability of $1-\mathrm{keV}$ electrons passing through a $\mathrm{C}_{60}$ sample in the gas. The collector aperture half-angle is $1.5^{\circ}$.

ponents present resonances around $6 \mathrm{eV}$, which lead to the excitation usually referred to as $\pi$, although the coupling with the $\sigma$ electrons in the plane is also important, as shown by the actual position of this peak in the EELS data discussed below. On the other hand, the broad peak of the imaginary part of the parallel component around $27 \mathrm{eV}$ reproduces correctly the loss spectrum of graphite. ${ }^{2}$ This correspondence between some polarizability features and EELS experiments reflects the fact that $\sigma$ electrons are almost uncoupled at energies above $10 \mathrm{eV}$ in graphite: the normal component of the polarization is very small, and the coupling between $\sigma$ electrons within a carbon plane vanishes due to the symmetry of the lattice (the coupling between atoms in different planes is also very weak due to the large separation between planes). Nevertheless, the surface curvature in carbon nanostructures breaks that symmetry, and, therefore, the spectra become sensitive to the geometrical shape of the

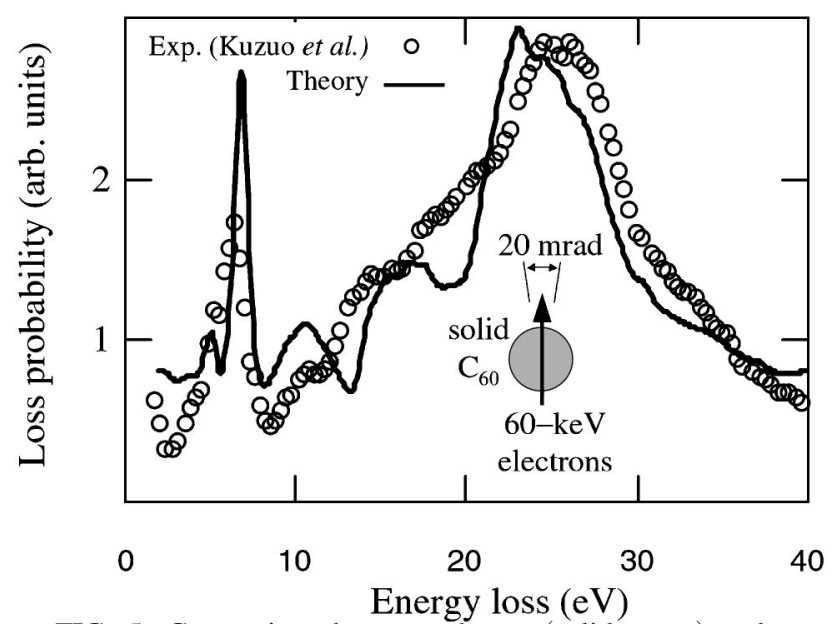

FIG. 5. Comparison between theory (solid curve) and experiment (circles, taken from Ref. 11) for the energy-loss probability of $60-\mathrm{keV}$ electrons in solid $\mathrm{C}_{60}$. The collector aperture half-angle (not specified in the experimental reference) has been set to 10 mrad. sample over distances larger than the interatomic separation.

Let us first study the energy-loss of an electron beam in a $(10,10)$ carbon nanotube to illustrate the case of an infinite atomic structure. ${ }^{20,38}$ The basic cell of this tube consists of a ring of diameter $1.37 \mathrm{~nm}$, formed by $40 \mathrm{C}$ atoms. This ring is periodically repeated with a period of $d=0.247 \mathrm{~nm}$ along the tube axis. For each $\mathrm{C}$ atom, the polarizability tensor has been built so that its normal axis is perpendicular to the plane formed by the three closest atoms. We have considered a typical experiment in transmission electron microscopy, where a $100-\mathrm{keV}$ electron beam is impinging normal to the cylinder axis. The screened interaction has been calculated in momentum space as shown in Sec. IV, and we work out the energy-loss probability per $\mathrm{C}$ atom by taking into account the contribution of neighboring cells to the sum of expression (12) (ten cells on each side of the central cell are enough to get good convergence above $3 \mathrm{eV}$ [see Fig. 2(b)]). Figure 2(a) shows the energy-loss probability for a typical value of the collector aperture of $\theta_{c}=1 \mathrm{mrad}$. The spectrum exhibits three features around 6.5, 11, and between 17-20 eV, which according to the analysis of Ref. 15 can be identified as $\pi$-surface plasmons and both low- and high-energy $(\sigma+\pi)$-surface plasmons, respectively. Below the $\pi$ feature, there is a shoulder in the loss spectrum that has been experimentally observed in both fullerenes s $^{3,11,12}$ and nanotubes. ${ }^{15,16}$ A comparison with Fig. 1 permits to postulate that these peaks derive from the coupling between the resonances of the $\pi$ and $\sigma$ electrons.

In Fig. 2(a), we have also plotted the contributions to the sum of Eq. (12) of the first neighboring cells with respect to a given central cell. Although the coupling between different cells involves atoms at different distances from each individual atom, these plots provide valuable information on the distance dependence of the electrodynamic interaction between atoms. First, one can see that the central cell and the first neighboring cells give contributions of the same order of magnitude. The strong cancellation between contributions from the central cell and the first neighbors at $\omega=5 \mathrm{eV}$ (i.e., the position of the sharp resonance in the normal component of the polarizability) proves the collective nature of $\pi$-plasmon resonances. This plot shows that the coupling between nearest cells gives rise almost entirely to the total energy-loss probability between $7 \mathrm{eV}$ and $17 \mathrm{eV}$, but more distant cells are needed outside this energy range. This point is relevant to the study of the $\sigma+\pi$ plasmon, which is around $24 \mathrm{eV}$ in fullerenes, whereas it shows up around 16 $\mathrm{eV}$ in nanotubes. As discussed above, this energy shift from fullerenes to nanotubes can be explained by the influence of curvature on the coupling between $\sigma$ orbitals. In Fig. 2(b), we plot the low-energy region of the same spectrum, where the contribution of more neighboring cells has been plotted separately. The $\pi$-plasmon peak is basically determined by coupling of the central cell with first neighbors, while the contribution of more distant cells overcomes that of first neighbors below this peak. This observation suggests that the position of the $\pi$-plasmon feature depends very little on the long-range structure of the sample, while the sub- $\pi$-plasmon features are more sensitive to geometry on a larger scale.

Reed and Sarikaya ${ }^{15}$ have measured loss spectra corre- 


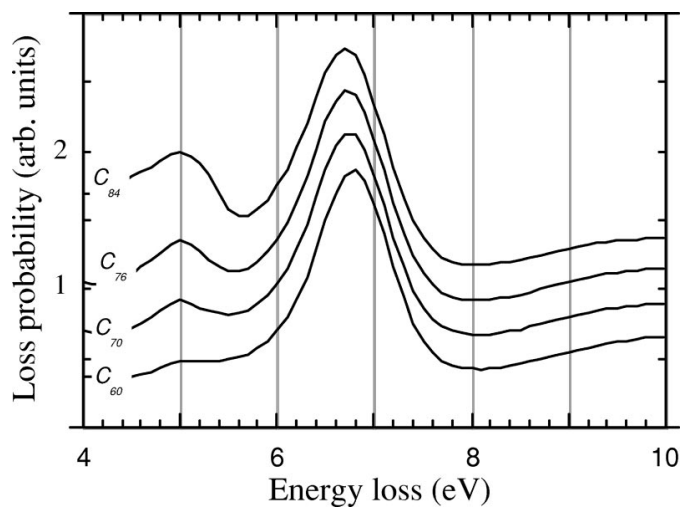

FIG. 6. Electron-energy-loss spectra calculated for different spherical carbon molecules. The plots have been shifted upwards to allow comparison. The primary electron energy is $60 \mathrm{keV}$. Electrons are collected up to a maximum scattering angle of $1 \mathrm{mrad}$.

sponding to a single nanotube of about $1.2 \mathrm{~nm}$ in diameter using a $100-\mathrm{keV}$ beam focused over a region of $0.2 \mathrm{~nm}$ in diameter. Their sample is similar to that used in our theoretical simulations. The spectrum corresponding to the probe passing through the tube presents a broad resonance around $17 \mathrm{eV}$, while a faint signature of a low-energy resonance is hidden by the elastic peak. For large impact parameters (several nanometers away from the tube axis), the low-energyloss peak becomes sharper and the position of the $(\sigma+\pi)$-plasmon feature is redshifted to $15 \mathrm{eV}$. Although the comparison of these data with those derived from our model is not straightforward because of the different type of beam, one can claim good qualitative agreement between them. The contribution of electrons with large impact parameter can be selected by collecting the electrons scattered within a small scattering angle. In Fig. 3(a) we show three spectra corresponding to different values of the collector aperture $\theta_{c}$. As the angle $\theta_{c}$ decreases, the $\pi$ peak becomes relatively higher, while the center of the $\sigma+\pi$ peak shifts down in energy. This dependence of the intensity of the different peaks of the spectrum on the scattering angle has been also experimentally confirmed for $\mathrm{C}_{60} \cdot{ }^{5} \mathrm{~A}$ simple explanation based on the classical EELS theory has been proposed by Reed and Sarikaya ${ }^{15}$ namely, that the impact-parameter dependence of the energy-loss probability goes like

$$
P(\omega) \sim e^{-2(\omega d / v)}, \quad d \geqslant v / \omega,
$$

where $d$ is the distance relative to a given scattering center. ${ }^{39}$ This means that distant collisions (i.e., small momentum transfers) excite low-energy modes more efficiently, a fact that explains the positive dispersion of the loss peaks. The analysis of the $\pi$-plasmon feature of Fig. 3(b) also shows a weak positive dispersion, which is in qualitative agreement with momentum-resolved EELS measurements in multishell fullerenes and peapods. ${ }^{10,16}$
The EELS simulation corresponding to spherical fullerenes is more straightforward, since the system has now a finite number of $\mathrm{C}$ atoms. ${ }^{40}$ In Fig. 4, we show the resulting computed loss spectrum in $\mathrm{C}_{60}$ as compared to experimental data reported by Keller and Coplan, ${ }^{5}$ measured in the gas phase, so that, as discussed in Sec. I, our simulation based upon a broad beam configuration suits best the experiment. The energy of the electrons is $1 \mathrm{keV}(v=8.6$ a.u.) and the collector aperture is $1.5^{\circ}$, which is small enough to justify the neglect of recoil, even at such a relatively low velocities. Although the theoretical spectrum seems to be shifted up in energy, the simulation reproduces reasonably well the relative positions of the peaks (including the small sub$\pi$-plasmon structure) as well as their relative intensities. In Fig. 5, we compare the results of our theory to the experiments of Kuzuo and co-workers ${ }^{12}$ for crystalline $\mathrm{C}_{60}$. The probe energy is now $60 \mathrm{keV}$ ( $v=61$ a.u.). We obtain good agreement in this case as well, suggesting that the coupling between neighboring target fullerenes is weak. This spectrum is very similar to those corresponding to $\mathrm{C}_{70}, \mathrm{C}_{76}$, and $\mathrm{C}_{84}$, with only small differences in the low-energy region below the $\pi$-plasmon feature, as shown in Fig. 6 . The $\pi$ plasmon around $6.7 \mathrm{eV}$ is very similar in all cases, except for a small redshift as the number of atoms in the molecule increases, in agreement with experiments using crystalline fullerenes, ${ }^{12}$ where the $\pi$ peak lies in the (6.4-6.1)-eV interval. (Nevertheless, one has to keep in mind that the interaction between neighboring fullerenes in the solid phase can play a role that is not described by the present calculations.) The almost identical shape of the $\pi$-plasmon peak in this series (Fig. 6) is in contrast with the strong differences at lower energylosses, as a consequence of the fact that the local atomic structure of all of these molecules is very similar up to first neighbors (which give the dominant contribution to the $\pi$ plasmon), while differences in geometrical ordering over larger distances play a significant role at lower energies.

In summary, we have obtained the screened interaction of a system of polarizable atoms to an external electric field, and have applied this function to study the energy-loss experienced by an electron (within a fully quantum-mechanical treatment of the probe) passing near carbon fullerenes and nanotubes. The spatial range of the coupling between dipoles explains the sensitivity of EELS to the structural details of the carbon nanostructures. The good agreement between theory and experiments is encouraging regarding the applicability of the derived screened interaction to other problems involving geometrically well-defined atomic structures.

\section{ACKNOWLEDGMENTS}

This work has been supported in part by the Basque Departamento de Educación, Universidades e Investigación, the University of the Basque Country UPV/EHU (Contract No. 00206.215-13639/2001), and the Spanish Ministerio de Ciencia y Tecnología (Contract No. MAT2001-0946).

\footnotetext{
*Email address: jga@sw.ehu.es

${ }^{1}$ H.W. Kroto, J.R. Heath, S.C. O'Brien, R.F. Curl, and R.E. Smalley, Nature (London) 318, 162 (1985).

${ }^{2}$ Y. Saito, H. Shinohara, and A. Ohshita, Jpn. J. Appl. Phys., Part 1
}

30, 1068 (1991).

${ }^{3}$ G. Gensterblum, J.J. Pireaux, P.A. Thiry, R. Caudano, J.P. Vigneron, Ph. Lambin, and A. Lucas, Phys. Rev. Lett. 67, 2171 (1991). 
${ }^{4}$ A. Lucas, G. Gensterblum, J.J. Pireaux, P.A. Thiry, R. Caudano, J.P. Vigneron, and Ph. Lambin, Phys. Rev. B 45, 13694 (1992).

${ }^{5}$ J.W. Keller and M.A. Coplan, Chem. Phys. Lett. 193, 89 (1992).

${ }^{6}$ D.A. Gorokhov, R.A. Suris, and V.V. Cheianov, Phys. Lett. A 223, 116 (1996).

${ }^{7}$ A.A. Lucas, L. Henrard, and Ph. Lambin, Phys. Rev. B 49, 2888 (1994).

${ }^{8}$ Dongmei Li, Steven Velasquez, and S.E. Schnatterly, Phys. Rev. B 49, 2969 (1994).

${ }^{9}$ M. Kociak, L. Henrard, O. Stephan, K. Suenaga, and C. Collieux, Phys. Rev. B 61, 13936 (2000)

${ }^{10}$ T. Pichler, M. Knupfer, M.S. Golden, J. Fink, and T. Cabioc'h, Phys. Rev. B 63, 155415 (2001).

${ }^{11}$ R. Kuzuo, M. Terauchi, M. Tanaka, Y. Saito, and H. Shinohara, Phys. Rev. B 49, 5054 (1994).

${ }^{12}$ R. Kuzuo, M. Terauchi, M. Tanaka, Y. Saito, and Y. Achiba, Phys. Rev. B 51, 11018 (1995).

${ }^{13}$ L.A. Bursill, P.A. Stadelmann, J.L. Peng, and S. Prawer, Phys. Rev. B 49, 2882 (1994).

${ }^{14}$ K. Suenaga, E. Sandré, C. Colliex, C.J. Pickard, H. Kataura, and S. Iijima, Phys. Rev. B 63, 165408 (2001).

${ }^{15}$ B.W. Reed and M. Sarikaya, Phys. Rev. B 64, 195404 (2001).

${ }^{16}$ X. Liu, T. Pichler, M. Knupfer, M.S. Golden, J. Fink, H. Kataura, Y. Achiba, K. Horahara, and S. Ijima, Phys. Rev. B 65, 045419 (2002).

${ }^{17}$ N. Van Giai and E. Lipparini, Z. Phys. D: At., Mol. Clusters 27, 193 (1993).

${ }^{18}$ P. Longe and S.M. Bose, Phys. Rev. B 48, 18239 (1993).

${ }^{19}$ A. Rubio, J.A. Alonso, J.M. López, and M.J. Scott, Physica B 183, 247 (1993).

${ }^{20}$ M.F. Lin and K.W.-K. Shung, Phys. Rev. B 50, 17744 (1994).

${ }^{21}$ L. Henrard and Ph. Lambin, J. Phys. B 29, 5127 (1996).

${ }^{22}$ U. Fano, Phys. Rev. 118, 451 (1960).
${ }^{23}$ A.A. Lucas and M. Sunjic, Prog. Surf. Sci. 2, 75 (1972)

${ }^{24}$ R.H. Ritchie, Philos. Mag. A 44, 931 (1981); R.H. Ritchie and A. Howie, ibid. 58, 753 (1988).

${ }^{25}$ A. Rivacoba, N. Zabala, and P.M. Echenique, Phys. Rev. Lett. 69, 3362 (1992); N. Zabala and A. Rivacoba, Phys. Rev. B 48, 14534 (1993); F.J. García de Abajo and A. Howie, Phys. Rev. Lett. 80, 5180 (1998).

${ }^{26}$ P. Moreau, N. Brun, C.A. Walsh, and H. Howie, Phys. Rev. B 56, 6774 (1997).

${ }^{27}$ J.U. Andersen and E. Bonderup, Eur. Phys. J. D 11, 435 (2000).

${ }^{28}$ Ch. Girard, Ph. Lambin, A. Dereux, and A.A. Lucas, Phys. Rev. B 49, 11425 (1994); P.A. Gravil, M. Devel, Ph. Lambin, X. Bouju, Ch. Girard, and A.A. Lucas, ibid. 53, 1622 (1996).

${ }^{29}$ B.E. Granger, Petr Král, H.R. Sadeghpour, and M. Shapiro, Phys. Rev. Lett. 89, 135506 (2002).

${ }^{30}$ T.L. Ferrell, R.J. Warmack, V.E. Anderson, and P.M. Echenique, Phys. Rev. B 35, 7365 (1987).

${ }^{31}$ A. Rivacoba, N. Zabala, and J. Aizpurua, Prog. Surf. Sci. 65, 1 (2000).

${ }^{32}$ D.R. Penn and P. Apell, J. Phys. C 16, 5729 (1983).

${ }^{33}$ P.M. Echenique, J. Bausells, and A. Rivacoba, Phys. Rev. B 35, 1521 (1987).

${ }^{34}$ F.J. García de Abajo, Scanning Microsc. 10, 9 (1996).

${ }^{35}$ R.G. Barrera and R. Fuchs, Phys. Rev. B 52, 3256 (1995).

${ }^{36}$ F.J. García de Abajo, Phys. Rev. Lett. 82, 2776 (1999); Phys. Rev. B 60, 6103 (1999).

${ }^{37}$ E.D. Palik, Handbook of Optical Constants of Solids (Academic Press, London, 1985).

${ }^{38}$ B.I. Dunlap, Phys. Rev. B 49, 5643 (1994).

${ }^{39}$ T.L. Ferrell and P.M. Echenique, Phys. Rev. Lett. 55, 1526 (1987).

${ }^{40}$ The structural data of fullerenes have been taken from http:// www.chem.sunysb.edu/msl/fullerene.html 